\title{
Reoperative innominate arterial, ascending aortic, and root replacement for extensive fungal endocarditis
}

\author{
Bradley G. Leshnower, MD, and Thomas G. Gleason, MD, Philadelphia, Pa
}

$\mathrm{F}$ Ungal endocarditis is a rare but lethal disease with a mortality rate exceeding $50 \%{ }^{1,2}$ The diagnosis is frequently elusive because the results of blood cultures are negative in $50 \%$ of cases. ${ }^{1,2}$ It is characterized by bulky and exophytic vegetations that typically emobolize. ${ }^{1}$ Prominent risk factors include anatomic cardiac abnormalities, previous valve operations, indwelling venous catheters, and the use of broadspectrum antibiotics. ${ }^{1,2}$ We report an unusual case of extensive Candida non-albicans endocarditis involving a mechanical valved conduit, ascending aortic graft, and innominate arterial graft 8 months after a complicated type A aortic dissection repair.

\section{Clinical Summary}

A 45-year-old man underwent a complicated repair of an acute type A aortic dissection, including a mechanical valved root and ascending aortic and innominate arterial replacement, at another institution. Eight months later, the patient presented with a temperature of $103^{\circ} \mathrm{F}$ and right ankle cellulitis surrounding a spider bite. Blood cultures grew Candida dubliniensis. Transesophageal echocardiography (TEE) demonstrated a small vegetation above the left coronary os. The patient was started on a regimen of caspofungin and transferred to our institution.

Repeat TEE confirmed the presence of the vegetation. The results of repeat blood cultures were negative. The patient was now afebrile and had no clinical signs or symptoms of endocarditis. He was discharged home receiving intravenous caspofungin therapy.

At his follow-up examination 4 weeks later, the patient remained afebrile and asymptomatic, and the results of blood cultures were still negative. Repeat TEE and computed tomographic angiography (CTA) demonstrated an interval increase in the number and size of the vegetations (Figures 1 and 2, A). He was started on a regimen of amphotericin B and underwent surgical exploration.

Opening of the ascending aortic graft revealed extensive and exophytic vegetations within the aortic root and ascending aortic and innominate arterial prosthetic grafts, with far more fungal burden seen than was predicted by either the TEE or CTA. All

From the Department of Surgery, Division of Cardiothoracic Surgery, University of Pennsylvania School of Medicine, Philadelphia, Pa.

Received for publication July 30, 2004; revisions received Aug 9, 2004; accepted for publication Aug 18, 2004.

Address for reprints: Thomas G. Gleason, MD, 3400 Spruce St, Silverstein 6, Division of Cardiothoracic Surgery, University of Pennsylvania School of Medicine, Philadelphia, PA 19103 (E-mail: thomas.gleason@ uphs.upenn.edu).

J Thorac Cardiovasc Surg 2005;129:941-2

$0022-5223 / \$ 30.00$

Copyright $\odot 2005$ by The American Association for Thoracic Surgery

doi:10.1016/j.jtcvs.2004.08.016 gross infection was confined to within the prosthetic grafts, with no invasion of the periannular structures. Immediate Gram staining revealed abundant budding yeast identified as Candida parapsilosis. All prosthetic graft, pledget material, and previously placed BioGlue (CryoLife, Inc, Kennesaw, Ga) were removed, and the arch was reconstructed during hypothermic circulatory arrest. The aortic graft, arch, and innominate arterial grafts were replaced with 28-mm and $12-\mathrm{mm}$ polyester grafts. A 23-mm Medtronic Freestyle porcine bioprosthesis (Medtronic, Inc, Minneapolis, Minn) was used to replace the aortic root after complete debridement.

The patient had an uneventful hospital course receiving intravenous amphotericin B and oral 5-flucytosine and was discharged on day 12 with a prescription for these drugs. After 4 months, the antifungal regimen was changed to daily oral fluconazole for long-term suppression. After more than 12 months of follow-up, there have been no signs or symptoms of endocarditis. Follow-up TEE and CTA at 3, 6, and 12 months demonstrated no evidence of recurrent endocarditis (Figure 2,B).

\section{Discussion}

Fungi are responsible for $1.3 \%$ to $6 \%$ of all cases of infective endocarditis. ${ }^{1,2}$ Most cases are caused by Candida albicans, but Candida parapsilosis is a more commonly isolated non-albicans species. Fungal pathogens create a biofilm, which aids in adherence to endothelial or prosthetic surfaces and prevents antifungal penetration, enabling persistent and recurrent infection. ${ }^{3}$ The high mortality rate of patients with fungal endocarditis correlates with a recurrence rate of $30 \%{ }^{1,2}$

An attempt at medical therapy is justified in patients with asymptomatic disease, particularly in the setting of reoperative cardiac surgery. ${ }^{4}$ However, medically treated patients require close follow-up, regardless of symptomatology. In this case surveillance TEE demonstrated an interval increase in the size and number of vegetations after 4 weeks of intravenous antifungal therapy, but neither TEE nor CTA depicted the true fungal burden, which extended throughout the prosthetic graft material.

A porcine bioprosthetic root was a good option for this case because the fungal burden was not invading the heart or the native vessels, the annulus was small given the patient's size, and the bioprosthetic root offered a better valvular hemodynamic profile than a mechanical valve of equal size. Although, historically, homograft replacement has been touted as the optimal strategy for reoperative endocarditis, neither late survival nor reoperation-free survival has been shown to be reduced by homograft use compared with use of other biologic or mechanical valves. ${ }^{5}$ Recurrences have been attributed to the discontinuation of long-term antifungal suppressive therapy and not to valve choice. ${ }^{6}$ Freestyle bioroots now appear to offer better 8-year freedom from structural deterioration and aortic insufficiency than homografts, ${ }^{7,8}$ and they might be a better long-term option when invasive infection is not present.

We conclude from this case that failed medical therapy, as demonstrated by TEE indicating a progression of the size, number, 

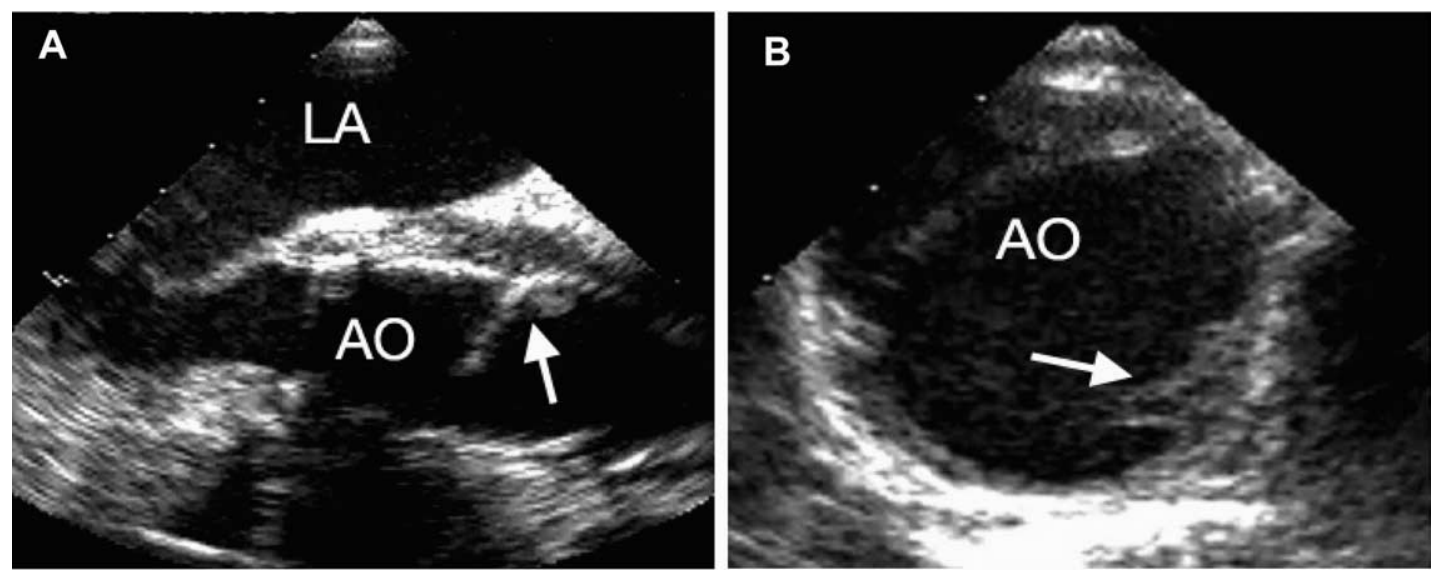

Figure 1. Preoperative TEE images of the aortic root in the long axis (A) and short axis (B) demonstrate endovascular fungal vegetations (arrows). The left atrium (LA) and aorta (AO) are labeled.
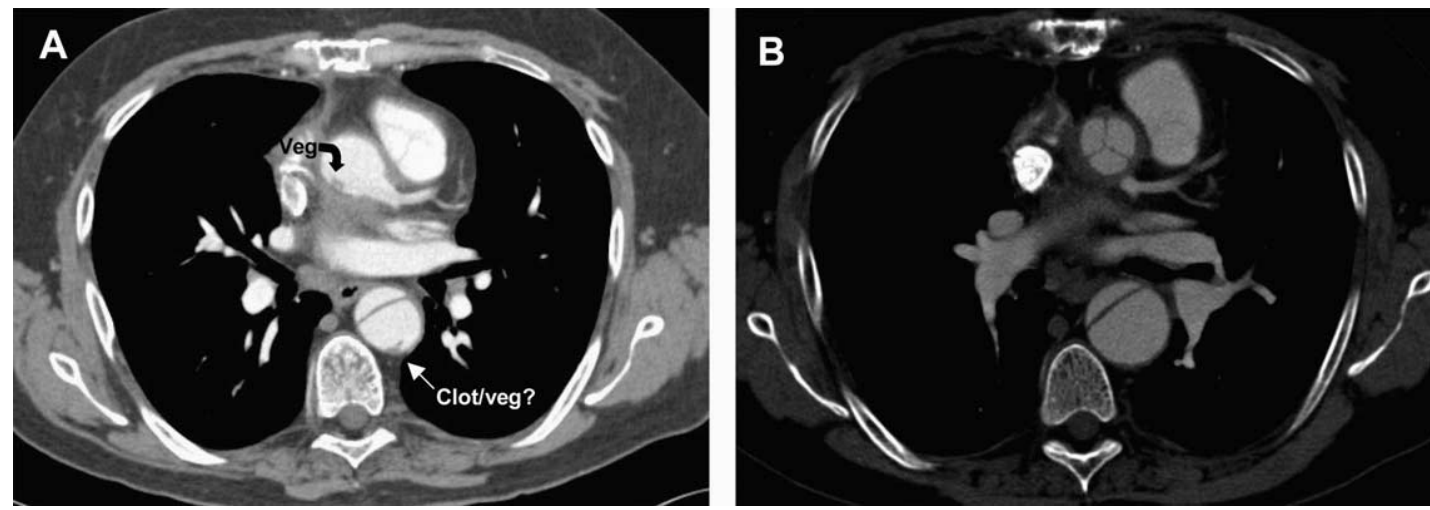

Figure 2. A, Preoperative multidetector helical CTA of the chest demonstrates endovascular vegetation near the left coronary os (veg). There is a small endovascular mass within the descending thoracic aorta that might represent either thrombus or vegetation (clot/veg?). Residual dissection is noted in the descending thoracic aorta. B, CTA 12 months postoperatively demonstrated no vegetation in the aorta or innominate arteries and complete resolution of the endovascular thrombus-vegetation in the descending thoracic artery. The porcine bioroot valve cusps are well visualized. The maximum diameter of the descending thoracic artery was noted to increase from $38 \mathrm{~mm}$ to $40 \mathrm{~mm}$ over the 12-month period.

or both of endocardial vegetations, can be a marker for more extensive endocardial fungal burden than is clinically apparent. This finding should prompt an aggressive multimodal strategy, including perioperative intravenous antifungal therapy, radical excision of infected prostheses and tissues, valve replacement, and long-term suppressive antifungal therapy. The patient described in this report continues to receive fluconazole and undergoes hepatic transaminase surveillance bimonthly and both TEE and CTA surveillance every 6 months.

\section{References}

1. Pierrotti LC, Baddour LM. Fungal endocarditis, 1995-2000. Chest. 2002;122:302-10.

2. Ellis ME, Al-Abdely H, Sandridge A, Greer W, Ventura W. Fungal endocarditis: evidence in the world literature, 1965-1995. Clin Infect Dis. 2001;32:50-62.
3. Weems JJ Jr. Candia parapsilosis: epidemiology, pathogenicity, clinical manifestations, and antimicrobial susceptibility. Clin Infect Dis. 1992; 14:756-66.

4. Zahid MA, Klotz SA, Hinton DR. Medical treatment of recurrent candidemia in a patient with probable Candida parapsilosis prosthetic valve endocarditis. Chest. 1994;105:1596-8.

5. Lytle BW, Priest BP, Taylor PC, Loop FD, Sapp SK, Stewart RW, et al. Surgical treatment of prosthetic valve endocarditis. $J$ Thorac Cardiovasc Surg. 1996;111:198-207.

6. Muehrcke DD, Lytle BW, Cosgrove DM 3rd. Surgical and long-term antifungal therapy for fungal prosthetic valve endocarditis. Ann Thorac Surg. 1995;60:538-43.

7. Kon ND, Riley RD, Adair SM, Kitzman DW, Cordell AR. Eight-year results of aortic root replacement with the freestyle stentless porcine aortic root bioprosthesis. Ann Thorac Surg. 2002;73:1817-21.

8. Takkenberg JJ, Eijkemans MJ, van Herwerden LA, Steyerberg EW, Lane MM, Elkins RC, et al. Prognosis after aortic root replacement with cryopreserved allografts in adults. Ann Thorac Surg. 2003;75: $1482-9$. 